\title{
Studies of HTO washout and deposition in the vicinity of emission source
}

\author{
A. Golubev, M. Khabibulin and S. Mavrin \\ Russian Federal Nuclear Center, VNIIEF, Mira Prospect 37, \\ 607190 Sarov, Nizhegorodsky Region, Russia
}

\begin{abstract}
There are presented in the repon research results of HTO wash-out and the model of HTO ammosphere concentration in the vicinity of a long-term HT and HTO emission source. The site of the scavenging experiments was around a $30 \mathrm{~m}$ emission source. The sampling arcs were chosen at $150-300 \mathrm{~m}$ from the base of the source to minimize dry deposition on the precipitation collectors. To study dependence of scavenging of tritium on raindrops characteristics, an optical device was constructed and used to measure the distribution of the drop radii and velocities during the period of experiment. The wash-out model, used for assessments, takes into account dispersion, deposition and re-emission. Soil surface is considered as secondary area source. Primary source is characterized as a source of infinite duration and permanent strength. Ingress of HTO to soil from primary source is occurred due to both dry and wet deposition. The model of HTO wet deposition is taken imto account kinetics of HTO exchange between vapor and liquid phase with parameters such as rain drop spectra, rain intensity, condensation-evaporation on drop's interface. Ingress of HT in soil is only due to HT dry deposition. Gauss type formulae for permanent emission source is used to calculate HTO atmosphere concentration. Averaged real meteorological data are used as input parameters for modeling.
\end{abstract}

\section{INTRODUCTION}

One of the key processes underlying environmental HTO propagation is HTO washout with precipitation from atmosphere. HTO interaction with water drops was studied both in laboratory and field (real-life) conditions. The paper [1] describes the laboratory experiments studying kinetics of exchange between the airflow containing deuterium oxide and water drops with no evaporation-condensation processes involved. The experiments performed were used to predict kinetics of HTO exchange with water drops. The paper [2] describes the experiments studying the rate of exchange with water drops flying through the atmosphere containing HTO. The paper [3] presents the kinetics model and identifies various phases of the said process. The experiments described in the paper [4] were conducted with the layout close to those in the papers $[2,3]$. The experiments involving researches into HTO exchange between atmosphere and water drops under field conditions are described in the paper [5]. No other publications concerning experiments in field conditions were presented. The current paper presents the results of field experiments and demonstrates that the model of irreversible HTO washout with raindrops yields overestimated results.

\section{Experiment description}

The experiment was performed under natural conditions in close vicinity to the emission source being at the height of 30 meters. The site around this source was divided into 36 sectors with the center in the source area. In each sector at the distance of 150 meters from the source, samplers with the volume of 0,5 liters were installed at the height of 1 meter from the soil surface. The distance of 150 meters from the source was selected due to the following factors. During long-term rains, the atmosphere is in neutral conditions corresponding to the D class stability and the wind velocity equal to $\sim 5 \mathrm{~m} \mathrm{~s}^{-1}$. For these atmospheric parameters, by using the Gauss model with Briggs parameters for urban conditions, the jet emitted from the stack was found to touch the ground surface at the distance of $\sim 100$ meters. Hence, 
sampling points will be on the initial site of the jet contact with the ground surface. At this distance, dry deposition arising due to the jet interaction with the ground surface will be negligible.

One of the major factors governing the rate of HTO washout from atmosphere with precipitation are rain parameters, i.e, drops' distribution in terms of size, velocity dependence of drops' falling on drops' diameter and rain intensity. To measure these parameters directly during the course of field experiments, a device to measure rain parameters was developed. Operation of this device is based on the photovoltaic technique. This technique allows concurrent and independent measuring of the diameter and fall velocity of drops. The device measures the infrared light beam due to raindrops flying through the volume measured. The pulse duration caused by the drop flying through the volume measured is determined by the velocity of a raindrop and the pulse amplitude is determined by the drop size. Each of the pulses is written into the memory of PC. By performing long-term measurements, one can measure rather a large number of drops and thereafter build a distribution of drops in terms of sizes and velocities.

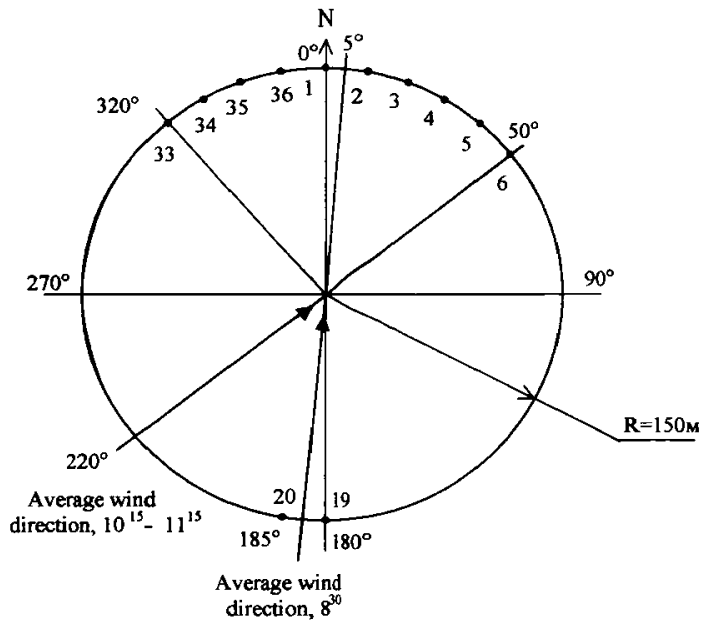

Rifure 1: The schematic of the experimert on November 17, 2000.

The experiment studying HTO washout from atmosphere with rain in field conditions was conducted on November 17,2000 . At 8.30, the average wind direction being of $185^{\circ}$ was determined and installing of samplers was started. Ten samplers were placed in the sector at $\pm 45^{\circ}$ from the average wind direction at sampling points from 6 to 33. Two samplers were installed on the leeward side at points 19 and 20 and were used as reference ones to estimate contamination caused by re-emission. At 10.15 it started raining. The experiment was over at 11.15. By that time $-20 \mathrm{ml}$ of water were collected in each sampler. During the experiment, the wind was varying its direction and by the end of the experiment its average direction was $220^{\circ}$. Fig. 1 presents the experiment layout. The meteodata and emission parameters during the course of the experiment are given in Table 1 .

HTO concentration in rainwater samples was measured with the Guardian scintillation counter. Table 2 presents the measurement results. 
Table 1. Meteodata and emission parameters in the experiment on November 17,2000 .

\begin{tabular}{|c|c|c|c|c|c|}
\hline $\begin{array}{c}\text { Time } \\
\text { (hours, minutes) }\end{array}$ & $\begin{array}{l}\text { Wind direction } \\
\text { (degrees) }\end{array}$ & $\begin{array}{l}\text { Wind velocity } \\
(\mathrm{m} / \mathrm{sec})\end{array}$ & $\begin{array}{c}\text { Humidity } \\
(\%)\end{array}$ & $\begin{array}{c}\text { Temperature, } \\
\left({ }^{\circ} \mathrm{C}\right)\end{array}$ & $\begin{array}{l}\text { HTO emission, } \\
\text { (relative units/min) }\end{array}$ \\
\hline $8: 30$ & $150-220$ & $\begin{array}{c}\text { Average }-5 \\
\text { Blasts up to } 10\end{array}$ & 93 & 5 & - \\
\hline $\begin{array}{c}\text { Commencement } \\
\text { of rain } \\
10: 15\end{array}$ & $200-240$ & $\begin{array}{l}\text { Average }-4 \text {, } \\
\text { Blasts up to } 8\end{array}$ & 93 & 5 & $2.44 \mathrm{E}+08$ \\
\hline $10: 30$ & $210-240$ & $\begin{array}{l}\text { Average }-4 \text {, } \\
\text { Blasts up to } 10\end{array}$ & 92 & 6 & $1.62 \mathrm{E}+08$ \\
\hline $10: 45$ & $230-260$ & $\begin{array}{l}\text { Average }-8 \\
\text { Blasts up to } 17\end{array}$ & 93 & $\overline{7}$ & $1.62 \mathrm{E}+08$ \\
\hline $11: 00$ & $210-240$ & $\begin{array}{c}\text { Average -4, } \\
\text { Blasts up to } 10\end{array}$ & 93 & 7 & $1.38 \mathrm{E}+08$ \\
\hline $\begin{array}{c}11: 15 \\
\text { experiment } \\
\text { completion }\end{array}$ & $210-240$ & $\begin{array}{c}\text { Average }-5 \\
\text { Blasts up to } 10\end{array}$ & 93 & 7 & $9.34 \mathrm{E}+07$ \\
\hline
\end{tabular}

Table 2. HTO concentration in rainwater samples.

\begin{tabular}{|l|c|c|c|c|c|c|c|c|c|c|c|}
\hline Sample & 1 & 2 & 3 & 4 & 5 & 6 & 36 & 34 & 33 & 19 & 20 \\
\hline Activity (relative units/l) & 12 & 110 & 480 & 3000 & 2900 & 4200 & 0.7 & 0.9 & 3.9 & 3.8 & 4.1 \\
\hline Error of activity measured (\%) & 5 & 2.4 & 5 & 0.5 & 0.5 & 0.4 & 6 & 10 & 8 & 4 & 7 \\
\hline
\end{tabular}

The average rain intensity during the sampling period was $1.42 \mathrm{~mm} \cdot$ hour $^{-l}$.

\section{WASHOUT MODEL}

Let us consider the fall of a raindrop through the HTO emission cloud. The coordinate axis " $z$ " coincides with the trajectory of the drop falling. The value $z=0$ corresponds to the ground level, $z_{\max }$ corresponds to the value on the axis with the HTO concentration in atmosphere being increased. Let the drop diameter be $D_{d}, \mathrm{~m}$; the falling velocity $-\mathrm{v}, \mathrm{m} \mathrm{s}^{-1}$. Distribution of the HTO concentration in atmosphere along the trajectory of drop fall can be written in the following way:

$$
\begin{aligned}
& C_{a x}(z)=C_{a x}\left(z_{\max } v t\right)=C_{a t}(t) \\
& z=\left(0 \div z_{\text {max }}\right), \quad t=\left(0 \div\left(z_{\max } / v\right)\right)
\end{aligned}
$$

where $z$ is a coordinate, $m$; $t$ is the current time of drop fall, $s ; C_{a z}(z)$ is HTO concentration distribution in atmosphere along the trajectory of drop fall, $\mathrm{Bq} / \mathrm{m}^{3} ; \mathrm{C}_{\mathrm{at}}(\mathrm{t})$ is the dependence of HTO concentration in atmosphere in the vicinity of the drop on time, $\mathrm{Bq} / \mathrm{m}^{3}$.

HTO concentration variation in he drop during its fall can be described by the following differential equation:

$$
\begin{gathered}
\mathrm{V}_{\mathrm{dr}} \frac{\mathrm{dC}_{\mathrm{dr}}(\mathrm{t})}{\mathrm{dt}}=\mathrm{f} \cdot \mathrm{S}_{\mathrm{dr} r}, \\
\mathrm{C}_{d r}(0)=\mathrm{C}_{0},
\end{gathered}
$$

where $V_{d r}$ - is the drop volume, $m^{3} ; f-$ is the flux density of HTO molecules at the air - drop surface interface, $\mathrm{Bq} /\left(\mathrm{m}^{2} \mathrm{c}\right) ; \mathrm{S}_{\mathrm{m}}-$ is the area of the drop surface, $\mathrm{m}^{2}, \mathrm{C}_{0}-$ is the initial HTO concentration in the drop, $\mathrm{Bq} / \mathrm{m}^{3}$

The density of HTO flux molecules was shown in the papers $[2,3]$ to have the following view:

$$
\mathrm{f}=\alpha\left(\mathrm{C}_{\mathrm{a}}-\gamma \cdot \mathrm{C}_{\mathrm{dr}} \cdot \mathrm{m}\right) \cdot(\mathrm{RT} / 2 \pi \mu)^{1 / 2},
$$


where $\mathbf{f}$ - is the resultant flux of HTO molecules at the interface drop/air, $\mathrm{Bq} /\left(\mathrm{m}^{2} \cdot \mathbf{s}\right) ; \alpha$-is the condensation coefficient depending on environmental conditions as well as on the aggregate state of water; $\mathrm{C}_{2}$ is the HTO concentration in surrounding air, $\mathrm{Bq} / \mathrm{m}^{3} ; \gamma$-is the coefficient of isotopic separation $\mathrm{H} / \mathrm{T}$, at $20^{\circ} \mathrm{C} \gamma=0.9 ; \mathrm{C}_{\mathbf{d}}-$ is the HTO concentration in liquid (drop), being in equilibrium with vapor $\mathrm{Bq} / \mathrm{m}^{3} ; \mathrm{R}=8,314$ joule/mole $\cdot \mathrm{K} ; \mathrm{m}$-is the content of saturated moisture in air, $\mathrm{m}^{3} / \mathrm{m}^{3} ; \mathrm{T}-$ is the temperature, $\mathrm{K} ; \mu$ - is the molecular mass, $\mathrm{kg} /$ mole. Depending on the sign, the expression (3) governs the rate of HTO molecules' evaporation and condensation. In [3] $\alpha$ was shown to be $3,4 \cdot 10^{-3}$ for HTO at $20^{\circ} \mathrm{C}$ and relative humidity of $90 \%$. By combining (2) and (3), one acquires the mathematical model of saturation for a single drop falling through the HTO emission cloud:

$$
\begin{gathered}
\frac{d C_{d r}(t)}{d t}=K_{1} \cdot\left[C_{d t}(t)-K_{2} \cdot C_{d r}(t)\right] \\
K_{1}=\frac{6 \cdot \alpha \cdot(R \cdot T)^{1 / 2}}{D_{d r} \cdot(2 \cdot \pi \cdot \mu)^{1 / 2}} \\
K_{2}=\gamma \cdot m \\
C_{d}(0)=C_{0} \\
t=\left(0 \div\left(z_{\max } / v\right)\right)
\end{gathered}
$$

Solution of equation (4) $-C_{d}(t)$ represents the specific variation of the activity of a raindrop during its fall. The specific activity of a raindrop fallen onto the ground surface is equal to the ratio of activity, contained in drops of all sizes, to the volume of these drops.

$$
C=\frac{\int_{0}^{\infty} C_{d r}\left(D_{d r}\right) \frac{\pi}{6} \cdot D_{d r}^{3} \cdot F^{\prime}\left(D_{d r}\right) d D_{d r}}{\int_{0}^{\infty} \frac{\pi}{6} D_{d r}^{3} \cdot F^{\prime}\left(D_{d r}\right) d D_{d r}}
$$

where $C$ - is the specific activity of rain water, $B q / \mathrm{m}^{3} ; C_{d r}\left(D_{d}\right)$ - is the specific activity of a $D_{d r}$, diameter raindrop fallen onto the surface, $\mathrm{Bq} / \mathrm{m}^{3} ; \quad F^{\prime}\left(\mathrm{D}_{\mathrm{dr}}\right)$ - is the share of drops with the size from $\mathrm{D}_{\mathrm{dr}}$ to $\left(D_{d}+d D_{d}\right) \cdot m^{-1}$.

The function $F^{\prime}\left(D_{d}\right)$ represents the derivative of the empirical Best formula [7]:

$$
F^{\prime}\left(D_{\mathrm{dr}}\right)=\left(\frac{D_{d r}}{A}\right)^{n} \cdot \frac{\mathbf{n}}{D_{d r}} \cdot \exp \left(-\left(\frac{D_{d r}}{A}\right)^{n}\right)
$$

where $n=2.25 ; \mathrm{A}$ - is the parameter depending on rain intensity [6]. [7]:

The rate of drops' fall depending on their sizes is described by the following empirical dependence

$$
v\left(D_{d r}\right)=4.874 \cdot D_{d r} \cdot \exp \left(-0.195 \cdot D_{d}\right)
$$

The modeling is implemented in the following sequence. Using the rain intensity measured in the experiment, the functions $F^{\prime}\left(D_{d}\right)$ and $v\left(D_{d r}\right)$ are determined by using the expressions $(6)$ and $(7)$. Then the integrals included into (5) are determined by the numerical method. In addition, for each drop size $D_{\text {tr }}$, the numerical differential equation is solved (4).

\section{RESULTS AND DISCUSSIONS}

According to the above washout model, the specific activity of rainwater is governed by the value of the condensation coefficient $\alpha$. Earlier this coefficient was determined in laboratory conditions [2,3]. The aim of the work was to verify possible application of this coefficient in conditions of real rain. To this end, the results of modeling and the experiment on November 17 were compared.

To determine HTO concentration in atmosphere, the Gauss method with Briggs parameters for urban conditions and the atmospheric stability class " $D$ " was used. Two peculiar features were taken into 
account when using this model for calculations. First, the variation in the average wind direction occurring during the experiment. Second, it was taken into account that rainwater sampling was conducted at the height of 1 meter above the ground surface, i.e, in the roughness zone. Inside the roughness zone, impurity dispersion becomes higher than at the height of the stack due to streamlining of various obstacles. When processing the experimental results, dispersion increasing in the roughness zone was taken into account by using indications of the weather vane placed in the roughness zone. To this end, the wind direction during each 15-minute interval was considered to be a random quantity distributed in accordance with the normal law. The interval, during which the wind direction was varying each 15 minutes, was taken to be $( \pm 2 \delta)$ in normal distribution. The average value (average of distribution) corresponded to the average wind direction during each of the 15 -minute intervals. Further on, the concentration above the sampler was determined as an average value (average of distribution) by using the following formula:

$$
C_{a t}=\int_{0}^{2 \pi} f(\varphi) F(\varphi) d \varphi,
$$

where $C_{a t}$ - is the HTO concentration in atmosphere above the sampler; $f(\varphi)$ is the HTO concentration in atmosphere calculated by using the Gauss formula for the wind direction $\varphi ; F(\varphi)$-is the function of probability density of normal distribution with the parameters $\varphi_{0}$, the average value of wind direction, and $\delta$, dispersion.

In the vicinity of the emission source there is some HTO concentration due to HTO re-emission by the ground surface. The re-emission affect was taken into the account in the following way. To HTO concentrations in rainwater obtained by using the models (4)-(7), the value of $3.95 \mathrm{E}+00$ relative units/iter was added. This value represents the arithmetical mean concentrations value of rainwater collected by samplers 19 and 20 . These samplers were installed on the leeward side. Therefore the contamination in them is caused primarily by HTO re-emission from the ground surface.

Fig. 2 represents computation comparison by using the models (4)-(7) and (8).

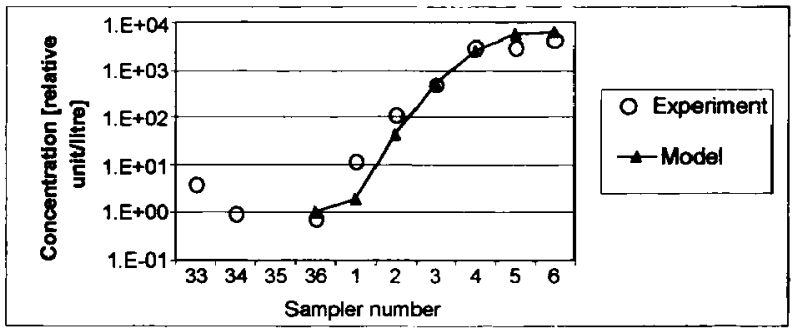

Deare 2: Comparison of the computations and the experiment

Lack of computational data at points 33 and 34 was caused by the fact that by the time of rain starting, the wind direction had changed and the jet did not flow above samplers 33 and 34 .

The results represented in fig. 2 shows that the washout model (4)-(7) using the condensation coefficient $\alpha$ acquired in laboratory experiments allows the description of field experiments. The madel, used to determine HTO concentration in atmosphere above the sampler, is a very essential component of the washout model. The modeling results show that the characteristic time of attaining the equilibrium concentration in a single raindrop is much lower than the characteristic time of flight of pollution cloud's drops. It leads to the fact that tritium concentration in the drop entered the sampler is determined by the 
HTO concentration in atmosphere in the vicinity of the ground surface rather than by the height-averaged HTO concentration in atmosphere.

\section{Acknowledgments}

The paper submitted was performed at RFNC-VNIIEF within the framework of ISTC Project 654.

The authors express their acknowledgement to the collaborators under the Project, namely to: Paula Tate (LLNL, USA) and Wolfgang Raskob (FZK, Germany), as well as their special appreciation of the contribution made by the Project Monitor Yves Belot (Independent Consultant, France) for arrangement of the experiments and discussion of the results.

\section{References}

[1].I. Friedman, L. Machta, R. Soller, J. Geophys. Res., Volume 67, 7, (1962) 2761-2766.

[2].L.F. Belovodsky, V.K. Gaevoy, T.N. Kosheleva, et.al., Sov. Atomic. Energy, Volume 52,4, (1982) 252-254.

[3].L.F. Belovodsky, V.K. Gaevoy, A.V. Golubev et al. J. Environ. Radioactivtivity, Volume 36, 2-3, (1997) 129-139.

[4].D.V. Booker, Quart. J. Roy. Meteorol. Soc., Volume 91, 73-79.

(Booker D.V., Exchange between water droplets and tritiated water vapor, Quart. J. Roy. Meteorol. Soc., v.91, p.73-79.)

[5].M.T.Dana, N.A. Wogman, M.A. Wolf. Atmospheric Environment, Volume 12, (1978)1523-1529.

[6].Khrian A.H. Fizika atmosferi. - M. State publishing house for physical and mathematical literature, $1958,476 \mathrm{pp}$.

[7].Pruppacher H. R. and Kellet J. D. Microphysics of clouds and precipitations D.Reidel Publishing Company, 1978, $714 \mathrm{p}$. 